\title{
STAT3 induces G9a to exacerbate HER3 expression for the survival of epidermal growth factor receptor-tyrosine kinase inhibitors in lung cancers
}

Yi-Fang Chang ${ }^{1,2,3+}$, Ken-Hong Lim ${ }^{1,2,3+}$, Ya-Wen Chiang ${ }^{2,3}$, Zong-Lin Sie ${ }^{4}$, Jungshan Chang ${ }^{5}$, Ai-Sheng Ho ${ }^{6}$ and Chun-Chia Cheng $^{7^{*}}$ (i)

\begin{abstract}
Background: HER3 mediates drug resistance against epidermal growth factor receptor (EGFR)-tyrosine kinase inhibitors (TKIs), resulting in tumor relapse in lung cancers. Previously, we demonstrated that EGFR induces HER3 overexpression, which facilitates the formation of cancer stem-like tumorspheres. However, the cellular mechanism through which EGFR regulates HER3 expression remains unclear. We hypothesized that EGFR downstream of STAT3 participates in HER3 expression because STAT3 contributes to cancer stemness and survival of EGFR-TKI resistant cancers.

Methods: First, RNAseq was used to uncover potential genes involved in the formation of lung cancer HCC827derived stem-like tumorspheres. EGFR-positive lung cancer cell lines, including HCC827, A549, and H1975, were individually treated with a panel containing 172 therapeutic agents targeting stem cell-associated genes to search for potential agents that could be applied against EGFR-positive lung cancers. In addition, gene knockdown and RNAseq were used to investigate molecular mechanisms through which STAT3 regulates tumor progression and the survival in lung cancer.

Results: BBI608, a STAT3 inhibitor, was a potential therapeutic agent that reduced the cell viability of EGFR-positive lung cancer cell lines. Notably, the inhibitory effects of BBI608 were similar with those associated with YM155, an ILF3 inhibitor. Both compounds reduced G9a-mediated HER3 expression. We also demonstrated that STAT3 upregulated G9a to silence miR-145-5p, which exacerbated HER3 expression in this study.

Conclusions: The present study revealed that BBl608 could eradicate EGFR-positive lung cancers and demonstrated that STAT3 enhanced the expression of HER3 through miR-145-5p repression by G9a, indicating that STAT3 is a reliable therapeutic target against EGFR-TKI-resistant lung cancers.
\end{abstract}

Keywords: BBI608, EGFR, G9a, HER3, Lung cancer, STAT3

\footnotetext{
* Correspondence: cccheng.biocompare@gmail.com

${ }^{\dagger}$ Yi-Fang Chang and Ken-Hong Lim contributed equally to this work.

${ }^{7}$ Radiation Biology Research Center, Institute for Radiological Research,

Chang Gung University / Chang Gung Memorial Hospital at Linkou, Taoyuan,

Taiwan

Full list of author information is available at the end of the article
}

(c) The Author(s). 2019 Open Access This article is distributed under the terms of the Creative Commons Attribution 4.0 International License (http://creativecommons.org/licenses/by/4.0/), which permits unrestricted use, distribution, and reproduction in any medium, provided you give appropriate credit to the original author(s) and the source, provide a link to the Creative Commons license, and indicate if changes were made. The Creative Commons Public Domain Dedication waiver (http://creativecommons.org/publicdomain/zero/1.0/) applies to the data made available in this article, unless otherwise stated. 


\section{Background}

The overexpression and activation of the epidermal growth factor receptor (EGFR), a transmembrane receptor tyrosine kinase that belongs to the ERBB family, facilitates tumor survival, proliferation, and cancer stemness in lung cancer [1, 2]. Therefore, EGFR-tyrosine kinase inhibitors (TKIs), such as gefitinib, afatinib, and osimertinib, specifically targeting EGFR wild-type (WT), EGFR and HER2 dual targets, and EGFR T790 M, respectively, are effective therapeutic agents for the eradication of lung cancers [3]. However, resistance to EGFR-TKIs still occurs and leads to tumor recurrence $[4,5]$.

Various mechanisms promote EGFR-TKI resistance. In addition to KRAS and EGFR T790 mutations, the expression of oncogenes, including MET [5-7], HER2 (ERBB2) [8], and epidermal growth factor receptor 3 (HER3, $E R B B 3)$ [5, 9, 10], is associated with drug resistance against EGFR-TKIs and leads to tumor recurrence in lung cancers. For example, long-term treatment leads to complement activation of the MET-mediated signaling pathway in lung HCC827 cells and consequent overexpression of HER3 against gefitinib [5]. Antitumor therapeutics by antibodies targeting HER3 triggers a response to EGFR-TKI erlotinib in refractory non-small-cell lung cancer [11]. Constitutive overexpression of HER2 forms dimerization with HER3, leading to the downstream activation of PI3K signaling and tumor survival $[12,13]$. Such results indicate that HER3 causes EGFR-TKI resistance. Therefore, it is vital to explore the molecular mechanism through which HER3 expression is regulated in EGFRpositive lung cancers.

We previously demonstrated that EGFR induces HER3 overexpression to promote the formation and survival of HCC827- and A549-derived cancer stem-like tumorspheres [14]. Because transducer and activator of transcription 3 (STAT3) contributes to cancer stemness [15, 16] and EGFR-TKI survival [17], we assume that STAT3 plays a major role in the regulation of HER3 expression. In addition, we revealed that EGFR phosphorylation participates in tumorsphere formation through the upregulation of the expression of G9a histone methyltransferase (HMT) [18]. Because YM155, an interleukin enhancerbinding factor 3 (ILF3) inhibitor [19], can block EGFR autophosphorylation to inhibit G9a-mediated stemness [18], EGFR downstream of G9a may also regulate cancer stemness and HER3 expression. Thus, the present study investigated the role of STAT3 and G9a in cancer stemness and HER3 expression.

G9a (EHMT2) has been reported to be an epigenetic regulator, which biochemically catalyzes the mono- and di-methylation of H3K9 (H3K9me1 and H3K9me2) in euchromatin [20], leading to gene repression [21]. Recently, G9a has been demonstrated to interact with several transcriptional factors, including GATA3 and ZEB2 [22],
STAT3 [16], and MYC [23], leading to the repression of gene transcription, while enhancing tumor survival. G9a is a potential mediator that silences tumor suppressors based on interacting partners. Particularly, G9a was reported to reduce the expression levels of microRNAs, such as miR200c, under the mediation of STAT3-G9a, which causes the astrocyte leptin receptor to exacerbate tumor progression in breast cancer [16]. In addition, G9a interacts with $\mathrm{MYC}$, which drives transcriptional repression and tumorigenesis [23]. To the best of our knowledge, STAT3 also induces MYC expression, and both transcriptional factors have been reported to participate in tumor stemness [24-27]. STAT3 was demonstrated as a target against EGFR-TKI resistance [21]. Therefore, we assumed that EGFR promotes HER3 overexpression through the STAT3-mediated activation of G9a to repress the expression of HER3-targeted microRNAs, because we identified that STAT3, an EGFR downstream phosphorylated target, participates in tumorsphere formation and survival in EGFR-positive colorectal cancer cell lines [15].

To validate the aforementioned assumptions, we used RNAseq to explore differential genes participating in the formation of HCC827-derived stem-like tumorspheres and in the knockdown of A549shSTAT3 and A549shG9a lung cancer cell lines compared with A549shLuc controls. The RNAseq-based gene profiling by treatment of BBI608, which selected by a screened panel targeting to stemassociated genes, and the inhibitory effects of BBI608 were compared with those of YM155, which particularly inhibited the formation of tumorspheres. BBI608, a STAT3 inhibitor, reduced not only the viability of EGFR-positive lung cancer cell lines but also the expression of G9a and HER3. These results were consistent with findings obtained following treatment with YM155, an ILF3 inhibitor. In addition, we demonstrated that STAT3 upregulated G9a expression, which significantly silenced miR-145-5p and exacerbated HER3 expression. The present study revealed the role of the transcriptional repression of miRNAs, such as miR-145-5p, by the STAT3-G9a axis in EGFR-positive lung cancers, which exacerbated HER3 expression and led to EGFR-TKI resistance.

\section{Methods}

\section{Cell culture and tumorsphere formation}

HCC827 (CRL-2868), A549 (CCL-185), H1975 (CRL5908), and H520 (HTB-182) lung cancer cell lines were purchased from the American Type Culture Collection (ATCC, Manassas, VA, USA). The cell lines were free from Mycoplasma. HCC827, H1975, and H520 cell lines were cultured in RPMI-1640 medium with 10\% fetal bovine serum (FBS) and 1\% penicillin-streptomycin. A549 was cultured in Dulbecco's modified Eagle medium with the same additives. The cell lines were reauthenticated through short tandem repeat profiling (Applied Biosystems, 
Massachusetts, USA): HCC827 on May 8, 2015; A549 on June 4, 2014; H1975 on May 23, 2019; H520 on December 13, 2016. For tumorsphere formation, cells were cultured in low-attached six-well plates with serum-free medium containing B27 (Invitrogen, Waltham, MA), $20 \mathrm{ng} / \mathrm{mL}$ of EGF (Sigma, Missouri, TX), $20 \mathrm{ng} / \mathrm{mL}$ of fibroblast growth factor (bFGF, Sigma), $5 \mu \mathrm{g} / \mathrm{mL}$ of bovine insulin (Sigma), and $4 \mu \mathrm{g} / \mathrm{mL}$ of heparin (Sigma) for at least a 7-day incubation period. The sizes of tumorspheres were examined under an inverted microscope (Axio Observer 3, ZEISS, Oberkochen, Germany). All cells were incubated at $37^{\circ} \mathrm{C}$ and $5 \% \mathrm{CO}_{2}$.

\section{Animals}

Male NOD/SCID mice were purchased from BioLASCO Taiwan Co., Ltd., Taiwan. Five-week-old mice were maintained under a 12 -h light/dark cycle at $22^{\circ} \mathrm{C}$. Animal studies were approved by the Institutional Ethical Review Committee at Mackay Memorial Hospital, Taiwan, and were performed according to NIH guidelines on the care and welfare of laboratory animals. Tumor xenografts were established by injecting $2 \times 10^{6}$ of A549shLuc $(n=4)$ or A549shSTAT3 $(n=4)$ into the subcutaneous legs of 5week-old mice. For tumor growth inhibition, $10 \mathrm{mg} / \mathrm{kg}$ of BBI608 was injected via tail vein in A549-derived tumor xenografts ( $n=3$ for each group). Tumors were externally measured using a digital caliper, and tumor volumes were calculated using the following formula: $0.52 \times$ width $^{2} \times$ length, where the smaller tumor diameter represented the width. Animals were sacrificed using carbon dioxide inhalation.

\section{RNAseq, small RNAseq profiling, and bioinformatics analysis}

RNAseq was performed to determine differentially expressed mRNAs in (1) HCC827-derived tumorspheres compared with parental HCC827 cells, (2) A549 cells treated with $1 \mu \mathrm{g} / \mathrm{mL}$ of BBI608 and $1 \mu \mathrm{g} / \mathrm{mL}$ of YM155, and (3) A549shSTAT3 and A549shG9a compared with A549shLuc (luciferase). A HiSeq 4000 with paired-end 150-bp sequencing was used for experiments. Genes upregulated with a $>2$-fold change $(\log 2)$ with a $p$ value of $<$ 0.05 in HCC827-derived tumorspheres were selected for bioinformatics analyses by using NetworkAnalyst (http:// www.networkanalyst.ca/) [28], and pathway activations were selected and matched based on the KEGG database. Genes downregulated with a less than -1-fold change $(\log 2)$ with a $p$ value of $<0.05$ in (1) BBI608- and YM155treated A549 cells compared with parental A549 cells and (2) A549shSTAT3 and A549shG9a compared with A549shLuc cells were compared using List Operations (http://www.molbiotools.com/listoperations.html) to determine common genes that were differentially expressed. In addition, differentially expressed genes were analyzed using NetworkAnalyst to determine major signaling pathways involved and key genes. Differentially expressed microRNAs were investigated using small RNA digitalization analysis through sequencing by synthesis (Illumina, San Diego, California, USA). The expression levels of known and unique miRNAs in each sample were statistically analyzed and normalized using transcripts per million clean tags (TPMs) [29]. Common differential miRNAs in A549shILF3 and A549shG9a identified using List Operations were compared with predictable HER3-binding miRNAs selected by TargetScan (http://www.targetscan.org/ vert_72/) based on conserved sites for broadly conserved miRNA families among vertebrates [30].

\section{Quantitative PCR}

The mRNA extraction and cDNA preparation were performed as described previously [18]. Quantitative PCR (Applied Biosystems, California, USA) was performed using the SYBR Green system (Applied Biosystems, California, USA) according to manufacturer's instructions. Primers used for PCR were as follows: ERBB3 (HER3): forward, 5'-GCCAATGAGTTCACCAGGAT-3' and reverse, 5'-ACGTGGCCGATTAAGTGTTC-3'. GAPDH: forward, 5' - GAGTCAACGGATTTGGTCGT-3' and reverse, 5' TTGATTTTGGAGGGATCTCG-3'.

\section{Gene knockdown and overexpression}

Gene knockdown was performed using a short-hairpin RNA (shRNA)-expression lentivirus system that contained the specific shRNA (target sequence of STAT3, ILF3, and EHMT2 (G9a): GCACAATCTACGAAGAATCAA, GCCATGTGATGGCAAAGCATT, and GCTCCAGGAA TTTAACAAGAT for shG9a\#1 / CGAGAGAGTTCATG GCTCTTT for shG9a\#2, respectively) in the pLKO.1-puro vector generated in a $293 \mathrm{~T}$ cell line. For G9a overexpression in A549 cells, pLenti6-MK1-EHMT2-V5 based on lentiviral system (Addegene, Massachusetts, USA) was purchased and used. The procedure followed was the same as that in our previous study [18].

\section{Western blot analysis}

Western blot analysis was performed as described previously [18]. Specific antibodies against ILF3, G9a, dimH3K9, STAT3, pSTAT3, HER3, and GAPDH were purchased from Cell Signaling (Danvers, Massachusetts, USA). Image J software was used to calculate the G9a, di-mH3K9, HER3 ratio divided by GAPDH.

\section{Cell viability}

The Alarmar Blue assay was performed according to manufacturer's instructions to determine cell viability. To find therapeutic agents that can be applied against EGFR-positive lung cancers, a panel containing 172 compounds targeting stemness (MCE, Monmouth 
Junction, NJ, USA) was added to HCC827, A549, H1975, and H520 cell lines separately at doses of $1 \mu \mathrm{M}$ and incubated for $48 \mathrm{~h}$. To examine cell viability, cells were treated using afatinib, BBI608, or YM155 for $48 \mathrm{~h}$.

\section{Cell migration}

Transwell migration assay $(8 \mu \mathrm{m})$ was used to detect A549 cell migration capacity. In brief, $5 \times 10^{4}$ cells were placed in the upper layer of a cell culture insert with $200 \mu \mathrm{L}$ of serum free DMEM medium. To test cell migration of A549-derived tumorspheres, serum-free DEME medium containing B27 (Invitrogen, Waltham, MA), $20 \mathrm{ng} / \mathrm{mL}$ of EGF (Sigma, Missouri, TX), $20 \mathrm{ng} / \mathrm{mL}$ of fibroblast growth factor (bFGF, Sigma), $5 \mu \mathrm{g} / \mathrm{mL}$ of bovine insulin (Sigma), and $4 \mu \mathrm{g} / \mathrm{mL}$ of heparin (Sigma) was used. Then, each $750 \mu \mathrm{L}$ of DMEM medium with $10 \% \mathrm{FBS}$ and test agents, including $1 \mu \mathrm{g} / \mathrm{mL}$ BBI608, 1 , $5,10 \mu \mathrm{g} / \mathrm{mL}$ UNC0642, was loaded into the below 24well culture plate. Cells were incubated at $37^{\circ} \mathrm{C}$ and $5 \%$ $\mathrm{CO}_{2}$ for $16 \mathrm{~h}$. The membrane inserts were fixed in $3.7 \%$ formaldehyde for $5 \mathrm{~min}$ and consequently incubated in $100 \%$ methanol for $20 \mathrm{~min}$ at room temperature. After $0.5 \%$ crystal violet in $2 \%$ ethanol to stain the membrane inserts for $15 \mathrm{~min}$ at room temperature, non-migrated cells on the upper membrane were scraped with cotton swabs. PBS wash for twice was necessary between operations. The cells migrated through the membrane were imaged and counted using an inverted microscope (Axio Observer 3, ZEISS, Oberkochen, Germany).

\section{Measurement of miR-145-5p}

A TaqMan advanced miRNA assay (Applied Biosystems, California, USA) was used to detect the expression of miR-145-5p in (1) lung cancer cell lines, including HCC827, A549, and H1975, (2) A549 cells treated with $1 \mu \mathrm{g} / \mathrm{mL}$ of BBI680 for $48 \mathrm{~h}$, and (3) A549shG9a with or without $20 \mathrm{ng} / \mathrm{mL}$ of EGF treatment in comparison with A549shLuc. The detection of miR-145-5p was according to manufacturer's instructions.

\section{Statistical analysis}

Statistical analyses were performed using GraphPad Prism v5.01 (GraphPad Software, Inc., California, USA). All analytical data with more than two groups were evaluated using analysis of variance, followed by post hoc analysis with Bonferroni's test. Student's $\mathrm{t}$ test was used to compare two groups. In addition, $p<0.05$ was considered to indicate a statistically significant difference.

\section{Results}

\section{HER3 overexpressed in HCC827-derived stem-like} tumorspheres

To validate that HER3 was overexpressed in lung cancer stem cells, we cultured lung cancer HCC827 cells in the serum-free medium described in Methods and Materials to develop an artificial stem-like cancer model (Fig. 1a), which expressed a high level of CD133, as demonstrated previously [18]. HER3 was overexpressed in HCC827derived tumorspheres analyzed through Western blot (Fig. 1b). Subsequently, RNAseq was used to find key genes involved in tumorsphere formation. The entire gene expression dynamics are presented in Additional file 1: Table S1. Based on NetworkAnalyst results and the matching of data based on KEGG datasets associated with pathways in cancers, KIT was identified, which is also a stem cell factor (Fig. 1c). In addition, KEGG searches linked to the ERBB signaling pathway validated HER3 (ERBB3) overexpression in tumorspheres (Fig. 1c). In addition, increased expression of genes belonging to the ERBB family, including EGFR, was detected by RNAseq, whereas the expression of CD133 (PROM1), a stem cell marker, significantly increased in HCC827 stem-like tumorspheres (Fig. 1d). To ensure that selected genes, namely ERBB2 and ERBB3, were oncogenes associated with lung cancer, a KaplanMeier plotter [31] was then used. According to results, the overexpression of ERBB2 and ERBB3 reduced the probability of survival of clinical patients with lung adenocarcinoma (Fig. 1e).

\section{BBI608, a STAT3 inhibitor, significantly reduced EGFR- positive lung cancers to against EGFR-TKI-resistance}

To find potential therapeutic agents against EGFRpositive lung cancers, particularly for reducing drug resistance associated with HER3, four lung cancer cell lines, namely EGFR-positive HCC827, A549, and H1975 cells and EGFR-negative H520 cells, were selected and investigated in the present study. The characteristics of the selected cell lines are illustrated in Fig. 2a. HCC827 is an EGFR E746-A750 deletion that is sensitive to EGFR-TKIs. A549 is an EGFR WT but with a KRAS mutation that is resistant to EGFR-TKIs. H1975 with an EGFR T790 M mutation also resists EGFR-TKI due to EGFR autophosphorylation. We observed that the expression of HER3 in EGFR-positive cell lines was higher than that in EGFR-negative H520 cells analyzed using qPCR (Fig. 2b). The results of Western blot were consistent and demonstrated high HER3 expression in H1975 cells that exhibited the simultaneous autophosphorylation of EGFR and increased phosphorylation of STAT3 than in other cell lines (Fig. 2c). In addition, a panel containing 172 therapeutic agents targeting genes associated with various stem cell pathways was used, and the viability of each cell line was measured after incubation with $1 \mu \mathrm{g} / \mathrm{mL}$ of therapeutic agents for $48 \mathrm{~h}$. The results are presented in Additional file 9: Figure S1. BBI608, a stemness inhibitor targeting the STAT3 pathway, significantly reduced selected EGFR-positive cell 


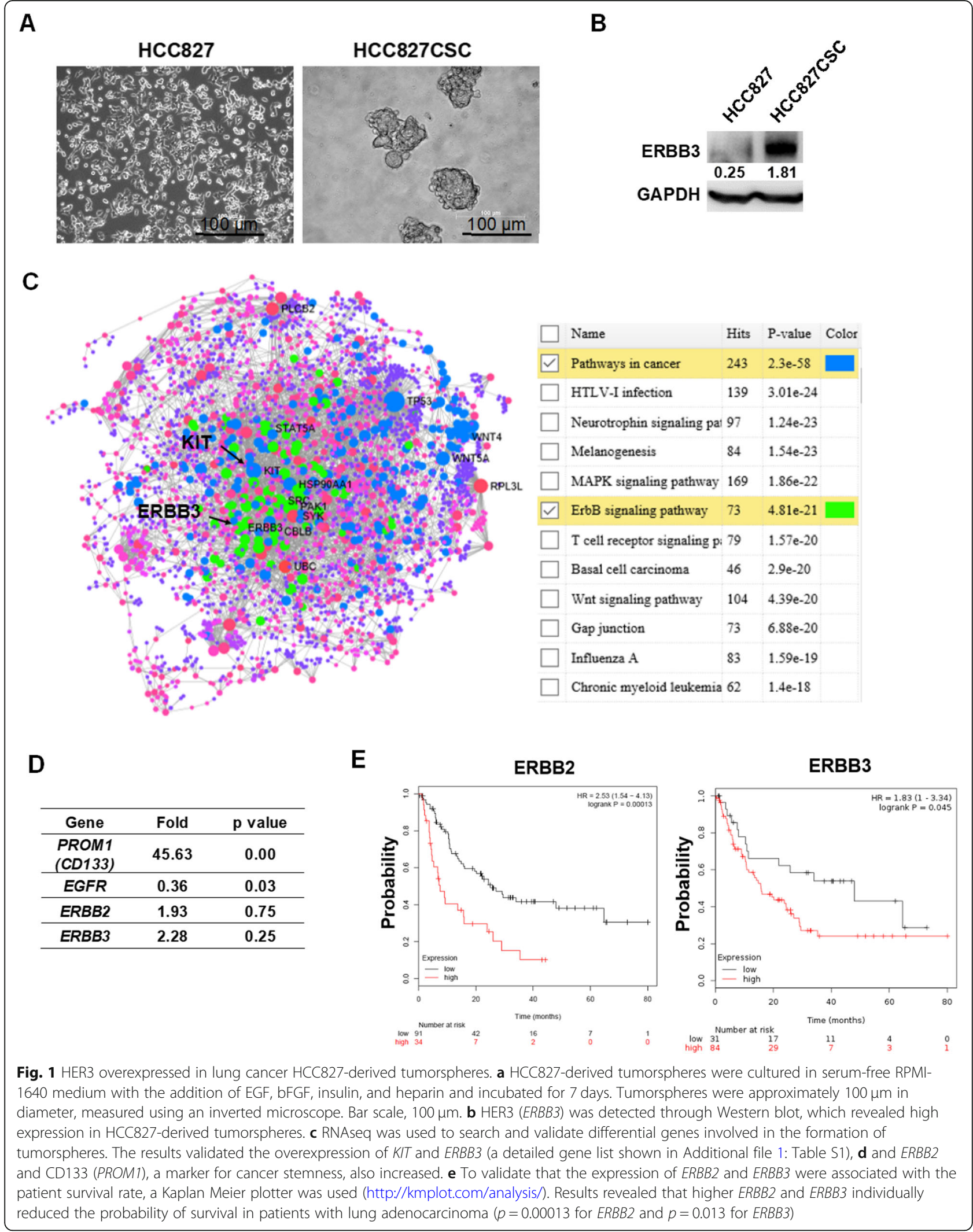




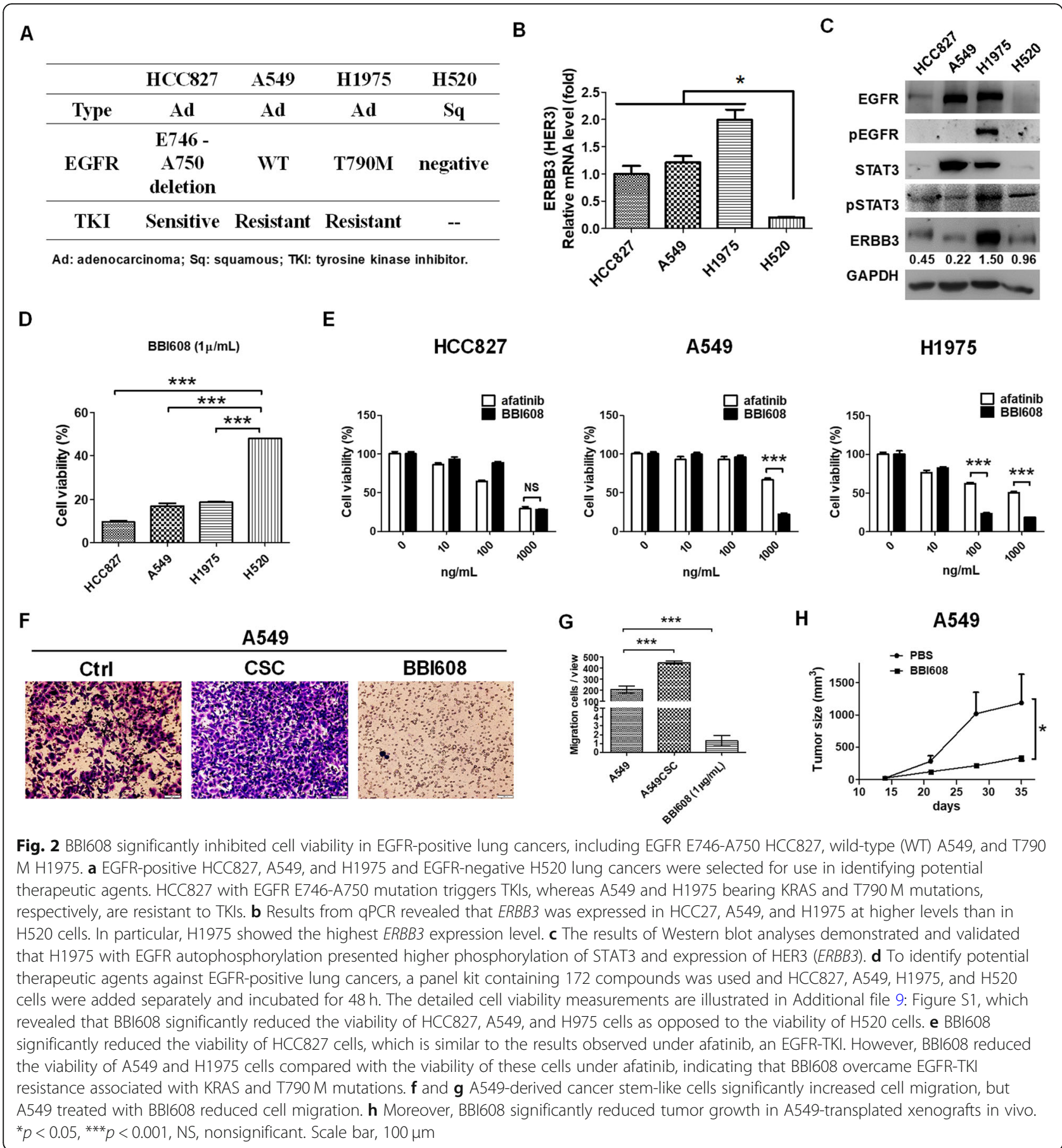

lines, with a cell viability less than $40 \%$. In addition, BIO targeting of CDK1 and GSK-3 reduced HCC827 and H1975 cell viability. Sanguinarine targeting of ERK particularly reduced A549 cell viability. The compound static targeting of STAT3 and halofuginone targeting of TGF $\beta /$ smad reduced cell viability both in A549 and H1975 cells. TG101209 targeting of JAKs specifically reduced cell viability against H1975 cells. The decrease in the viability of HCC827, A549, and H1975 cells following treatment with BBI608 was validated, and the viability of cell lines was lower than that of H520 cells (Fig. 2d). In addition, there was no significant difference between the decrease in cell viability caused by afatinib and BBI608 in HCC827 cells; however, BBI608 caused a substantial reduction in the viability of both A549 and H1975 cells (Fig. 2e), indicating that STAT3, the BBI608 
target gene, mediated EGFR-TKI resistance. We also found that cell migration capacity was increased in A549 cells-derived cancer stem-like cells in vitro, which was reduced in $1 \mu \mathrm{g} / \mathrm{mL}$ of BBI608 treatment (Fig. $2 \mathrm{f}$ and g). We found BBI608 was able to induce cell apoptosis in vitro (Additional file 12: Figure S4). BBI608 also inhibited tumor growth in A549-derived tumor xenografts in vivo (Fig. 2h). The results revealed that BBI608 was a potential therapeutic agent against lung cancer.

\section{STAT3 contributes to G9a and HER3 expression and} influences cell survival in HCC827-derived tumorspheres

To validate that STAT3 was a therapeutic target against lung cancer stem cells, HCC827-derived tumorspheres were incubated with $1 \mu \mathrm{g} / \mathrm{mL}$ of afatinib, BBI608, and YM155, and the diameters of tumorspheres were measured. In our previous studies, YM155, an ILF3 inhibitor, was demonstrated to be an efficient agent against the formation of cancer stem-like tumorspheres and HER3 expression [14, 18]. As expected, BBI608 and YM155 significantly reduced tumorsphere formation, whereas afatinib had no effect (Fig. 3a and b). Subsequently, STAT3 was knocked down in A549 cells, and results revealed a reduction in HER3 expression in A549shSTAT3 cells compared with A549shLuc cells (Fig. 3c). Because both BBI608 and YM155 inhibited tumorsphere formation in vitro, the capacities of the two therapeutic agents to alleviate inhibition were measured against A549 cells. Results revealed that BBI60 and YM155 had similar inhibitory effects in the $0-10 \mu \mathrm{g} / \mathrm{mL}$ concentration range (Fig. 3d), which implied that the two compounds had a similar inhibitory target. The transcriptomic profiling analysis based on RNAseq revealed consistent gene expressional clusters in BBI608- and YM155-treated A549 cells (Fig. 3e). Differentially expressed genes are listed in Additional file 2: Table S2 for BBI608-treated A549 cells and in Additional file 3: Table S3 for YM155-treated A549 cells. A total of 309 downregulated genes were common between BBI608 and YM155 treatments (Fig. 3f and Additional file 6: Table S6); these genes were associated with pathway with cancer and the ERBB signaling pathway, which included ERBB3 (HER3) and BIRC5 (Survivin) (Fig. 3g), whereas BIRC5 was previously identified as an inhibitory gene of YM155 [32]. In addition, $E R B B 2$ decreased following treatment with the two inhibitors (Fig. 3g). These results suggest that STAT3 was a target of YM155. Therefore, we investigated STAT3 phosphorylation and G9a expression in BBI608 and YM155 treatments with or without EGF co-treatment in A549 cells, whereas G9a was demonstrated as a downregulated target of YM155 [18]. Both BBI608 and YM155 reduced the endogenous and EGF-induced phosphorylation of STAT3, resulting in decreases in G9a and HER3 expression (Fig. 3h).
G9a mediated STAT3-regulated HER3 expression in EGFRpositive lung cancer

Because the expression of both G9a and HER3 decreased following treatment with BBI608 and YM155, we investigated whether G9a regulated the expression of HER3 in lung cancer. First, we validated that the knockdown of STAT3 reduced the viability of lung cancer cells significantly (Fig. 4a), which is consistent with the results of our previous study that demonstrated that STAT3 facilitated the survival of colorectal cancer stem-like cells [15]. In addition, there was a significant reduction of tumor growth in vivo in the A549shSTAT3 cell line compared with the A549shLuc cell line based on a tumor xenograft model (Fig. 4b and c). However, knockdown of G9a did not affect in vitro cell viability in A549 cells and in vivo A549induced tumor xenografts (Additional file 10: Figure S2). The transcriptomic profiles of A549shSTAT3 and A549shG9a cell lines analyzed using RNAseq were compared with A549shLuc (Additional file 4: Table S4 for A549shSTAT3 and Additional file 5: Table S5 for A549shG9a). There were 245 commonly downregulated genes between A549shSTAT3 and A549shG9a cell lines (Fig. 4d and Additional file 6: Table S6), including $E R B B 3$, which was observed in the NetworkAnalyst analysis results (Fig. 4e). However, ERBB2 expression decreased only in the A549shSTAT3 cell line. In addition, there were 55 common genes between 309 and 245 genes from the BBI608/YM155 and the shSTAT3/shG9a axis, respectively (Additional file 11: Figure S3 and Additional file 6: Table S6). The results indicated the suppression of the ERBB signaling pathway, which particularly inhibited HER3 expression. To further ensure that G9a was capable of inducing HER3 expression, qPCR and Western blot analyses were performed to investigate HER3 expression in EGF-treated A549shG9a cell lines and H1975 cell lines treated with G9a inhibitors, including UNC0642 and BIX01294. Results showed a considerable decrease in HER3 expression in EGF-treated A549shG9a cell lines compared with EGF-treated A549shLuc cell lines (Fig. 4f). In addition, EGFmediated STAT3 phosphorylation increased HER3 expression in a time-dependent manner, which was inhibited in A549shG9a cell lines without influencing EGF-mediated STAT3 phosphorylation (Fig. 4g). Moreover, UNC0642 and BIX01294 inhibited the expression of HER3 in a dose-dependent manner, whereas H3K9 dimethylation was a target of G9a, which was also inhibited by UNC0642 and BIX01294 (Fig. 4h). Overexpression of G9a led to increase of HER3 in A549 cells (Fig. 4i), suggesting that G9a contributed to the overexpression of HER3 in EGFRpositive lung cancers. 


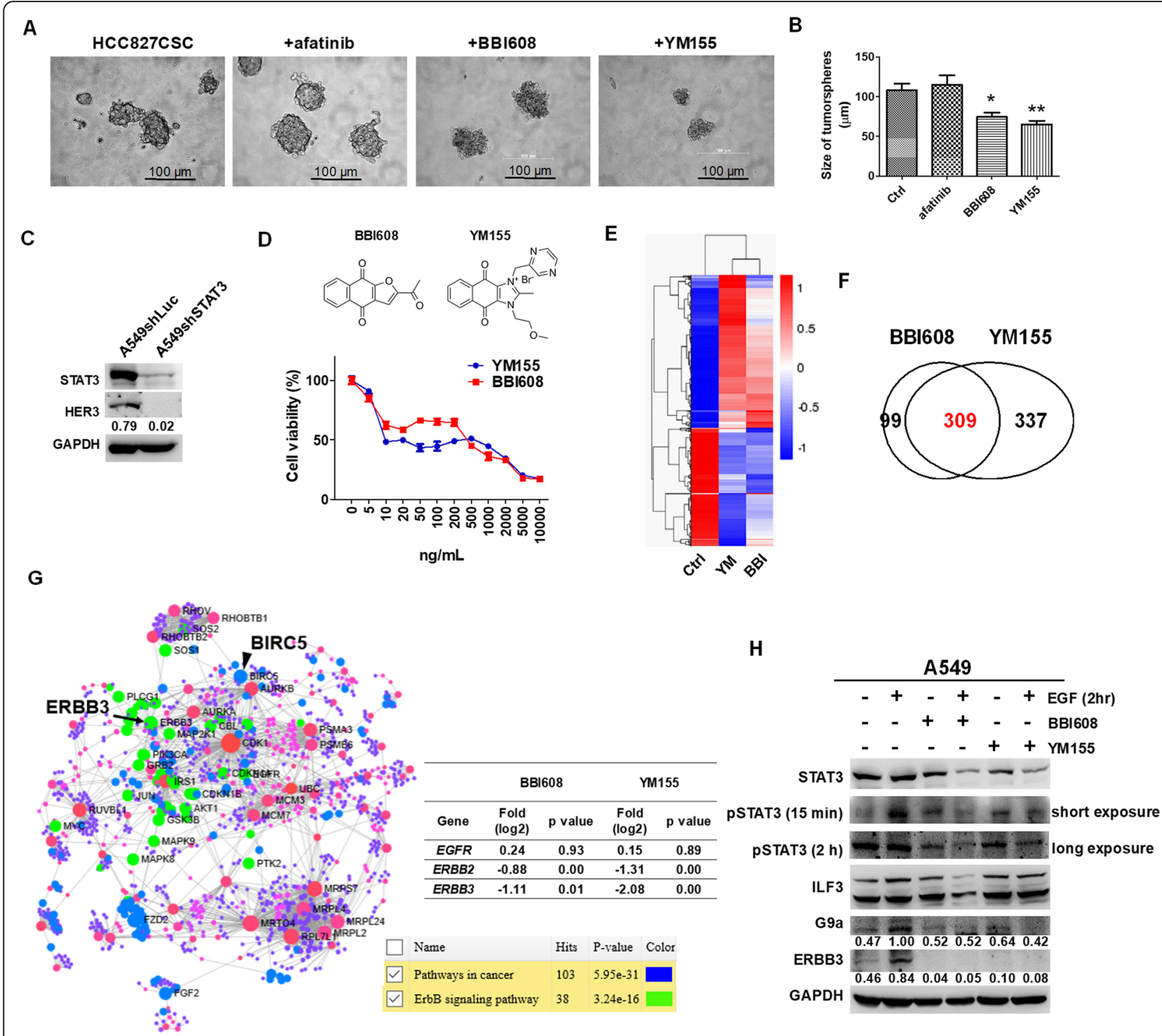

Fig. 3 BBI608 and YM155 inhibited STAT3 phosphorylation and G9a and ERBB3 expression in EGFR-positive lung cancers. a To validate the inhibitory capacity of BB1608 and YM155 against the formation of cancer stemness, HCC827-derived stem-like cancer tumorspheres (HCC827CSC) were treated separately with $1 \mathrm{\mu g} / \mathrm{mL}$ of afatinib, BBI608, and YM155 over a 7-day incubation. We demonstrated that BBI608 and YM155 could reduce the formation of HCC827-derived tumorspheres compared with afatinib as observed under an inverted microscope. $\mathbf{b}$ The tumorsphere sizes of HCC827CSC were measured and compared. Results showed that BBI608 and YM155 significantly reduced HCC827CSC formation, whereas afatinib exhibited no effect at a concentration of $1 \mu \mathrm{g} / \mathrm{mL}$. c There were lower levels of HER3 in A549shSTAT3 compared with A549shLuc, indicating that STAT3 facilitated HER3 expression. $\mathbf{d}$ In addition, BBI608 and YM155 caused a similar reduction in the viability of A549 cells in a dose-dependent manner. e Gene cluster profiling from the RNAseq analysis revealed a similar pattern between BBI608-and YM155-treated A549 cells (Additional file 2: Table S2 and Additional file 3: Table S3). f There were 309 common differentially expressed genes between BBI608- and YM155-treated A549 cells (Additional file 6: Table S6), g which were consequently analyzed by NetworkAnalyst to identify key potential genes mediated by BBI608 and YM155. Results revealed and validated reductions in ERBB2 and ERBB3 expression, whereas BIRC5 is an inhibitory target of YM155. $\mathbf{h}$ In addition, BB1608 and YM155 substantially reduced endogenous and EGF-mediated STAT3 phosphorylation, resulting in G9a and HER3 (ERBB3) reductions. ${ }^{*} p<0.05,{ }^{* *} p<0.01$. Scale bar, $100 \mu \mathrm{m}$

\section{G9a repressed miR-145-5p and exacerbated HER3 expression}

To investigate the potential mechanism through which G9a regulated the expression of HER3, we investigated whether microRNAs were regulated and inhibited by G9a, because G9a is an HMT and has a gene silencing function. A549shILF3 and A549shG9a cell lines with a low HER3 expression level (Fig. 5a) were selected for application in investigating miRNA expression profiles by using small RNAseq. We observed that the knockdown of ILF3 reduced G9a expression levels (Fig. 5a). The gene cluster profiles of A549shILF3 and A549shG9a cell 


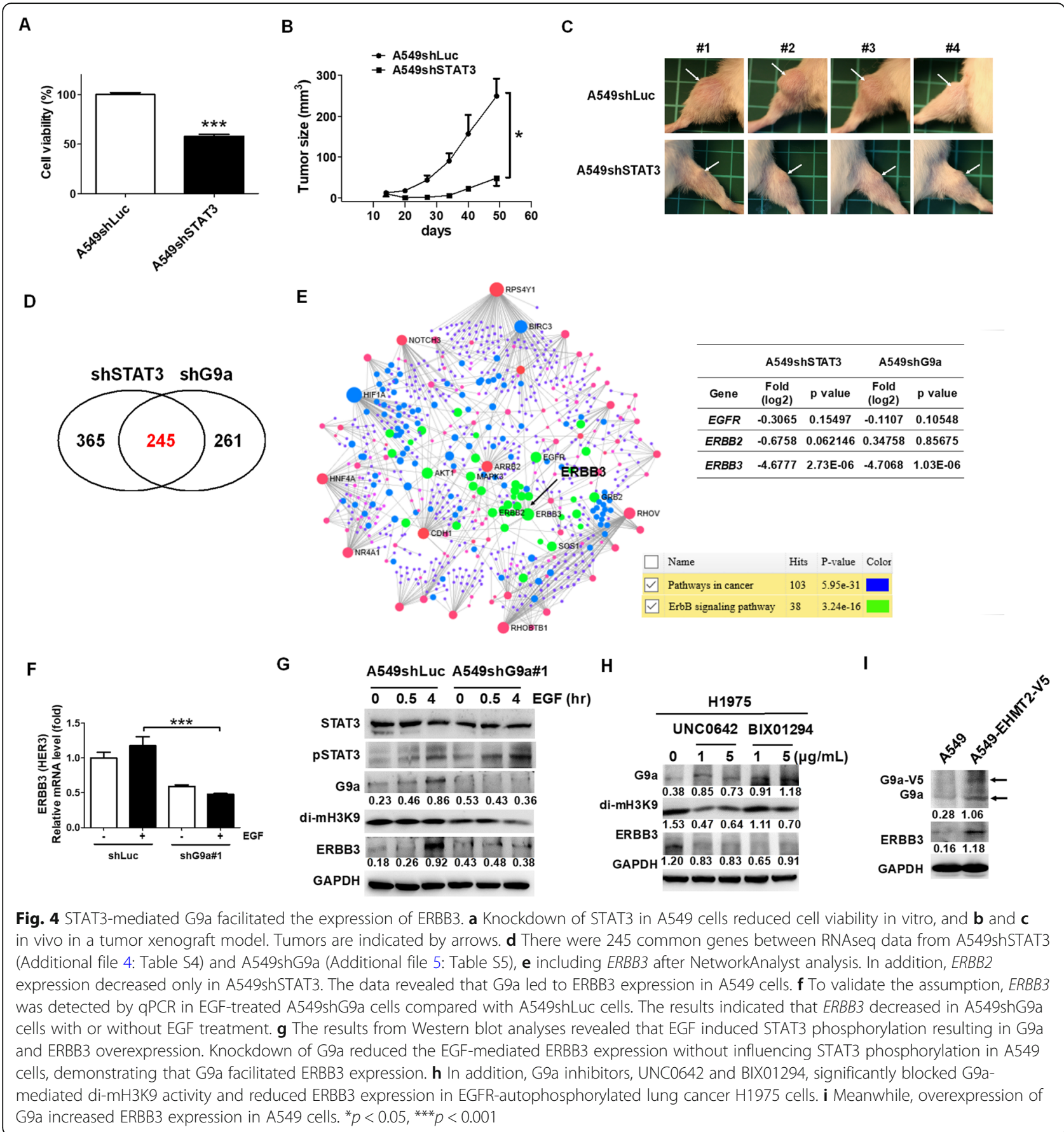

lines are illustrated in Fig. 5b, which indicated there were 124 upregulated and 75 downregulated miRNAs in the A549shILF3 cell line (Additional file 7: Table S7), and 62 and 48 miRNAs were upregulated and downregulated, respectively, in the A549shG9a cell line compared with the A549shLuc cell line (Additional file 8: Table S8) (Fig. 5c). Among increased miRNAs, there were 24 miRNAs commonly expressed between A549ILF3 and A549shG9a cell lines, which were further compared with HER3-targeted miRNAs predicted using
TargetScan. We identified three miRNAs that were also predicted as HER3-targeted miRNAs, namely miR-130a3p, miR-145-5p, and miR-19-3p (Fig. 5d). A study revealed that miR-145 is capable of targeting and inhibiting HER3 in breast cancer [33]. We found 4 genes regulated by STAT3-G9a-miR-145-5p through comparing the 55 common gene (Additional file 11: Figure S3A) with miR-145-5p-targeted gene from TargetScan, including ERBB3 (HER3), PLA2GA4, MTUS1, TSKU (Additional file 11: Figure S3C). Therefore, miRNA- 


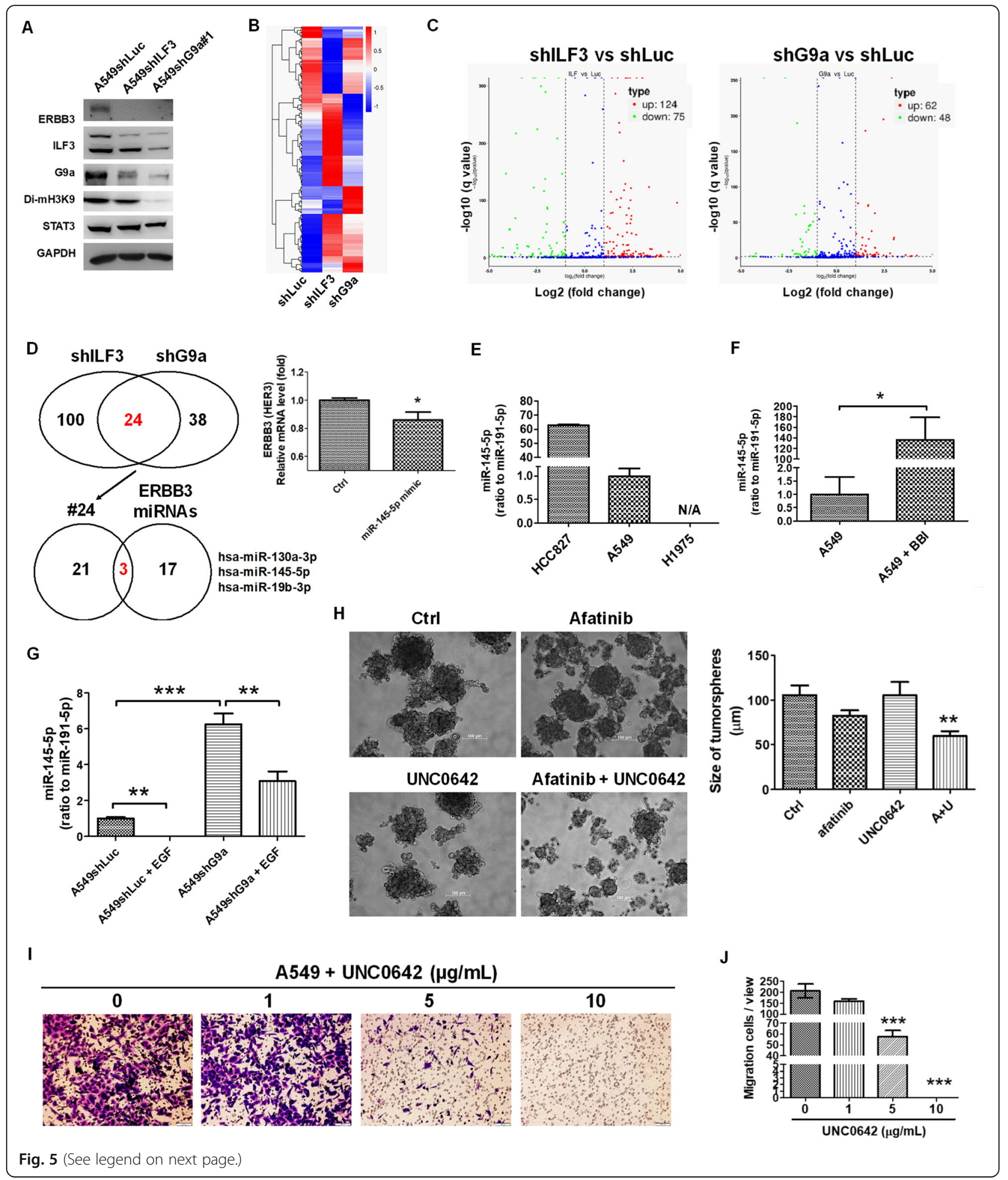


(See figure on previous page.)

Fig. 5 G9a induced ERBB3 expression via the repression of miR-145-5p. a Western blot analysis revealed that the knockdown of ILF3 and G9a led to a reduction in HER3 (ERBB3) expression in A549 cells. There was a reduction of G9a in A549ILF3 cells, whereas demethylation of H3K9 presented G9a activity. b The gene cluster profiles in A549shILF3 and A549shG9a were listed and compared following small RNAseq analysis, c which revealed that 124 and 75 miRNAs were upregulated and downregulated, respectively, in A549shILF3 (Additional file 7: Table S7), and 62 and 48 miRNAs were upregulated and downregulated, respectively, in A549shG9a cells compared with A549shLuc cells (Additional file 8: Table S8). $\mathbf{d}$ There were 24 miRNAs with increased expression that were common between A549ILF3 and A549shG9a, and 3 potential miRNAs involved in HER3 expression, which were compared with the HER3-targeted miRNAs from TargetScan, including miR-130a-3p, miR-145-5p, and miR-19-3p. In addition, miRNA-145-5p transfection into A549 cells reduced HER3 expression detected by qPCR. e The expression of miRNA-145-5p in HCC827 cells was higher than in A549 and H1975 cells. f BBl608 (1 $\mu \mathrm{g} / \mathrm{mL})$ treatment significantly increased miR-145-5p expression in A549 cells. g In addition, the miRNA-145-5p level significantly increased in A549shG9a compared with in A549shLuc cells, which reduced in EGF treatment. $\mathbf{h}$ Therefore, co-treatment of afatinib with UNC0642 significantly reduced HCC827-derived tumorsphere formation based on tumorsphere diameter. $\mathbf{i}$ and $\mathbf{j}$ Therefore, targeting G9a by UNC0642 also reduced cell migration in A549 cells in a dose-dependent manner. ${ }^{*} p<0.05,{ }^{* *} p<0.01,{ }^{* * *} p<$ 0.001. Scale bar, $100 \mu \mathrm{m}$

145-5p was transfected into A549 cells, which resulted in a decrease in the HER3 expression detected by qPCR analysis (Fig. 5d). In addition, miRNA-145$5 p$ was expressed highly in the HCC827 cell line compared with the expression level in A549 and H1975 cell lines (Fig. 5e). miRNA-145-5p expression was negatively correlated with the expression of HER3, as illustrated in Fig. 2b. In addition, BBI608 significantly increased miR-145-5p expression (Fig. 5f). miRNA-145-5p expression levels increased significantly in A549shG9a cells compared with in A549shLuc cells, which reduced following treatment with EGF (Fig. 5g). The results demonstrated that STAT3-G9a increased HER3 expression through the repression of miR-145-5p. We then demonstrated that co-treatment of afatinib with UNC0642 significantly reduced the formation of HCC827-derived tumorspheres measured based on tumorsphere diameters (Fig. 5h), which is consistent with the findings following treatment with BBI608 and YM155 (Fig. 3a and b). In addition, UNC0642 targeting G9a reduced cell migration of A549 cells in a dose-dependent manner (Fig. 5i and $\mathrm{j}$ ) and induced A549 cell apoptosis (Additional file 12: Figure S4).

\section{Discussion}

The present study demonstrated that STAT3 promotes HER3 expression through the activation of G9a, which, in turn, represses HER3-targeted miR-145-5p expression in EGFR-positive lung cancers (Fig. 6). We identified a potential therapeutic agent, BBI608, which targeted STAT3, and significantly and effectively reduced cell proliferation and tumorsphere formation in EGFRpositive cell lines. In addition, BBI608 efficiently inhibited G9a-exacerbated HER3 expression. Because HER3 facilitates EGFR-TKI resistance, our results indicate that the STAT3 blockade is a promising strategy that can be employed to eradicate EGFR-TKI-resistant lung cancers, including KRAS-mutant A549 and T790 M-mutant H1975 cells.

We previously demonstrated higher EGFR phosphorylation in HCC827-derived stem-like tumorspheres even without EGF treatment [18]; therefore, in the present model, the activation of STAT3 was considerable. Compared with HCC827 with an EGFR E746-A750 deletion, there was higher STAT3 phosphorylation and HER3 expression in H1975 cells (Fig. 3c), indicating that EGFR autophosphorylation led to the subsequent cascades.

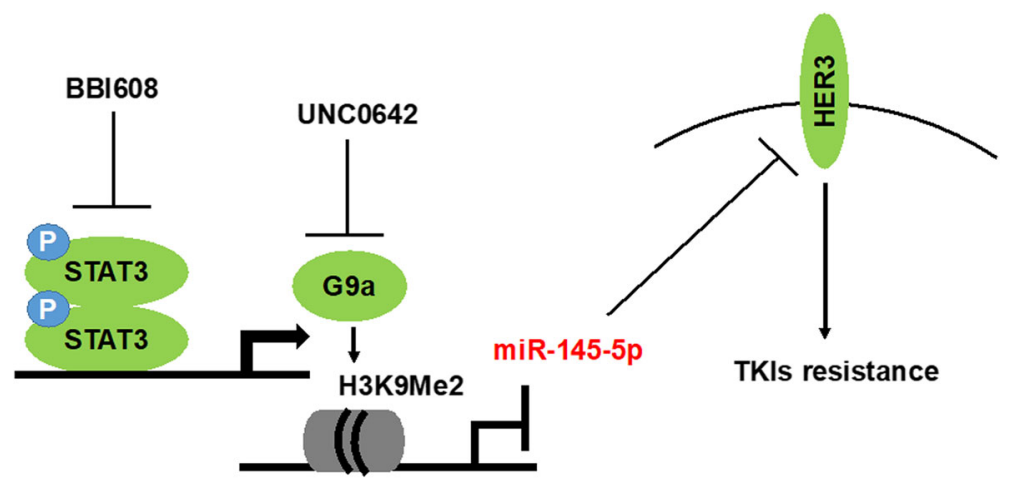

Lung cancer

Cancer stem-like tumorsphere

Fig. 6 A proposed model illustrating the STAT3-G9a-miR-145-5p axis as a therapeutic target that regulates HER3 expression governing TKIs resistance in lung cancer-derived stem like-tumorspheres 
Previous studies have revealed that the activation of STAT3 is not only achieved by EGFR [17] but also by IL6-gp130 [34, 35]. Particularly, the inhibition of STAT3 phosphorylation increased the inhibitory effects of afatinib, an EGFR-TKI, in T790-mutant H1975 cells [34]. Therefore, targeting STAT3 is considered a potential strategy for overcoming EGFR-TKI resistance associated with KRAS and T790 M mutations in lung cancers. In addition, because we demonstrated that STAT3 induced HER3 expression in EGFR-positive lung cancers, the inhibition of STAT3 could prevent HER3-mediated EGFRTKI resistance. Previous studies have indicated that the amplification of MET participates in EGFR-TKI resistance, which could be associated with MET-mediated HER3 expression [5] and MET-mediated AKT and MAPK signaling pathways [36]. A combined strategy simultaneously targeting STAT3 and MET could effectively prevent HER3-derived EGFR-TKI resistance.

In clinical trials, STAT3 inhibition has been reported to have remarkable therapeutic effects against various cancer types. For example, OPB-51822, a small-molecule STAT3 phosphorylation inhibitor targeting the SH2 domain (Tyr705/Ser727), exhibited high antitumor activity in EGFR-TKI-resistant NSCLC patients on a first-inman phase I study [37]. In addition, STAT3-targeted RNAi therapies, such as AZD9150 (16-oligonucleotide antisense molecule targeting the $3^{\prime}$ untranslated part of STAT3), also exhibited single-agent antitumor activity in patients with lymphoma or NSCLC in a phase I dose escalation study [38]. However, the STAT3-HER3 cascade is a source of EGFR-TKI resistance. The amplification of oncogenes, such as HER2, also results in EGFR-TKI resistance [8]. In addition, previous studies have revealed that cancer stem cells that exhibit self-renewal and pluripotency are responsible for drug resistance and cancer recurrence [39]. The present study revealed that HER2 was overexpressed in HCC827-derived tumorspheres (Fig. 1d), and HER2 expression decreased following treatment with BBI608 (Fig. 3g) and A549shSTAT3 (Fig. 4e). Because there was no change in the expression of HER2 in A549shG9a (Fig. 4e), we concluded that STAT3 was a potential therapeutic target against lung cancers with EGFR-TKI resistance that was superior to G9a.

Increasing evidence suggests that microRNAs are involved in EGFR-mediated signaling pathways in lung cancers, including miR-145, which is downregulated and associated with TKI resistance targeting ERK, AKT, Oct4, c-MYC, EGFR, and NUDT1 [40]. The results indicate that miR-145 is a cell proliferation suppressor in lung adenocarcinoma by targeting EGFR and NUDT1 as an ERK and AKT phosphorylation inhibitor, which enhances gefitinib cytotoxicity in NSCLC [41, 42]. We further demonstrated that miR-145 was inhibited by G9a in the present study; thus, it played a major role in the regulation of HER3 expression. Because miR-145 has been reported to be a suppressor of HER3 translation in breast cancer [33], miR-145 downregulation by G9a could promote the overexpression of HER3 in lung cancers. In addition, we observed the downregulation of HER3 in A549shILF3 cells compared with A549shLuc cells, which validated that ILF3 enhanced HER3 expression [14]. A previous study reported that IL3 mediates miR-145 biogenesis and enhances the development of cancer stem cells in bladder cancer [43]. miR-145 targets EGFR [41], resulting in the downregulation of EGFR in A549ILF3 cells [14]. Therefore, YM155, an ILF3 inhibitor, caused a substantial decrease in EGFR [7], which led to the decrease in EGF-mediated G9a levels in the present study. Similarly, we confirmed that miR-200c, which is highly expressed and associated with epithelialmesenchymal transition, invasion, and migration in NSCLC patients [44], was inhibited by G9a in A459 cells measured based on small RNAseq analyses, indicating that G9a not only regulated HER3 through the repression of miR-145-5p expression but also increased other oncogenes, such as the astrocyte leptin receptor, through the repression of miR-200c expression, which enhanced tumorigenesis in lung cancers.

\section{Conclusions}

In conclusion, we demonstrated the STAT3-G9a-HER3 axis in lung cancer that evades EGFR-TKI therapies. The potential mechanism is through the repression of miR145-5p expression, which specifically targets HER3 (Fig. 6). BBI608 and YM155 demonstrated similar effects, reducing the viability of lung cancer A549 cells and inhibiting STAT3-mediated G9a and HER3 expression. We propose that BBI608, which was selected from a panel kit, and YM155 are potential therapeutic agents against EGFR-positive lung cancers and may be combined with EGFR-TKIs for application in the eradication of lung cancers.

\section{Supplementary information}

Supplementary information accompanies this paper at https://doi.org/10. 1186/s12885-019-6217-9.

Additional file 1: Table S1. Differential genes in the HCC827-derived tumorspheres analyzed by RNAseq. (XLSX 1505 kb)

Additional file 2: Table S2. Differential genes in the BBI608-treated A549 cells analyzed by RNAseq. (XLSX 105 kb)

Additional file 3: Table S3. Differential genes in the YM155-treated A549 cells analyzed by RNAseq. (XLSX $160 \mathrm{~kb}$ )

Additional file 4: Table S4. Differential genes in the A549shSTAT3 cells analyzed by RNAseq. (XLSX 109 kb)

Additional file 5: Table S5. Differential genes in the A549shG9a cells analyzed by RNAseq. (XLSX 113 kb) 
Additional file 6: Table S6. The decreased 309 genes overlapping between BBI608- and YM155-treated A549 cells, and decreased 245 genes overlapping between A549shSTAT3 and A549shG9a cells. (XLSX 15 kb)

Additional file 7: Table S7. Differential miRNAs in the A549shILF3 cells analyzed by small RNAseq. (XLSX $22 \mathrm{~kb}$ )

Additional file 8: Table S8. Differential miRNAs in the A549shG9a cells analyzed by small RNAseq. (XLSX $16 \mathrm{~kb}$ )

Additional file 9: Figure S1. BBI608 is a potential therapeutic agent against lung cancers. A panel kit containing 172 compounds was used to search for therapies effective against EGFR-positive HCC827, A549, H1975, and EGFR-negative $\mathrm{H} 520$ cell lines. The effective agents were selected based on a cell viability level lower than $40 \%$. Among the therapies, only BBI608 markedly reduced cell viability against HCC827, A549, and H1975 rather than $\mathrm{H} 520$ cells. (TIF $2396 \mathrm{~kb}$ )

Additional file 10: Figure S2. Knockdown of G9a did not affect cell viability in A549 cells and in A549-derived tumor xenografts. (A) G9a was knockdowned using shRNA techniques, that did not reduce cell viability in A549 cells, and (B) in a A549-derived tumor xenograft model. NS, no significant. (TIF $145 \mathrm{~kb}$ )

Additional file 11: Figure S3. There were 55 reduction genes among BBI608 (BBI)-, YM155 (YM)-, shSTAT3, and shG9a-treated A549 cells (Additional file 6: Table S6), which were subsequently analyzed using NetworkAnalyst. (A) The 55 genes were classified using PANTHER (http://www. pantherdb.org/) based on molecular functions. The genes were listed based on their molecular functions, including binding (24 genes), catalytic activity (16 genes), molecular function regulator (5 genes), molecular transducer activity (3 genes), structural molecule activity (1 gene), transcription regulator activity (2 genes), and transporter activity. (B) NetworkAnalyst revealed that the ERBB signaling pathway was the major inhibitory pathway, particularly reducing ERBB3 expression. (C) STAT3-G9aregulated genes were compared with miR-145-5p-targeted genes from TargetScan resulted in four overlapping genes, including PLA2G4A, MTUS1, ERBB3, and TSKU. (TIF $912 \mathrm{~kb}$ )

Additional file 12: Figure S4. BBI608 and UNC0642 led to apoptosis in A549 cells. Apoptosis was detected in BBl608- and UNC0642-treated A549 cells using a flow cytometry, whereas FL1-H and FL2-H represented annexin-FITC and propidium iodide staining, respectively. Results indicate that BBI608 and UNC0642 caused A549 apoptosis from 1.06 to $45.08 \%$ by $1 \mu \mathrm{g} / \mathrm{mL}$ concentration and from 0.17 to $11.85 \%$ by $10 \mu \mathrm{g} / \mathrm{mL}$ concentration, respectively, for $4 \mathrm{~h}$ incubation. (TIF $396 \mathrm{~kb}$ )

\section{Abbreviations}

EGFR: Epidermal growth factor receptor; FBS: Fetal bovine serum; ILF3: Interleukin enhancer-binding factor 3; shRNA: Short-hairpin RNA; STAT3: Transducer and activator of transcription 3; TKIs: Tyrosine kinase inhibitors; TPMs: Transcripts per million clean tags

\section{Acknowledgments}

The authors thank the Radiation Biology Core Laboratory of Institute for Radiological Research, Chang Gung Memorial Hospital, for technical support. This manuscript was edited by Wallace Academic Editing.

\section{Authors' contributions}

CCC and YFC conceptualized and designed the study. CCC and ASH developed the methodology. CCC and YWC collected the data. CCC and JC analyzed and interpreted the data (e.g., statistical analysis, biostatistics, and computational analysis). CCC, KHL, ZLS and YFC contributed toward writing, reviewing, and revising the manuscript. YFC and KHL supervised the study. All authors read and approved the final manuscript.

\section{Funding}

This study was supported by grants from the Ministry of Science and Technology of Taiwan (MOST 106-2320-B195-003), Cheng Hsin General Hospital (CHGH 106-06), and Mackay Memorial Hospital (MMH-CT-10605 and $\mathrm{MMH}-106-61)$. Funding bodies did not have any influence in the design of the study and data collection, analysis and interpretation of data or in writing the manuscript.

\section{Availability of data and materials}

All data generated or analyzed during this study are included in this published article and its Additional files.

\section{Ethics approval and consent to participate}

Animal studies were approved by the Institutional Ethical Review Committee at Mackay Memorial Hospital, Taiwan, and were performed according to NIH guidelines on the care and welfare of laboratory animals.

\section{Consent for publication}

Not applicable.

\section{Competing interests}

The authors declare that they have no competing interests.

\section{Author details}

'Division of Hematology and Oncology, Department of Internal Medicine, Mackay Memorial Hospital, Taipei, Taiwan. ${ }^{2}$ Laboratory of Good Clinical Research Center, Department of Medical Research, Mackay Memorial Hospital, Tamsui District, New Taipei City, Taiwan. ${ }^{3}$ Department of Medicine, Mackay Medical College, New Taipei City, Taiwan. ${ }^{4}$ Institute of Molecular and Genomic Medicine, National Health Research Institute, Miaoli, Taiwan. ${ }^{5}$ Graduate Institute of Medical Sciences, School of Medicine, College of Medicine, Taipei Medical University, Taipei, Taiwan. ${ }^{6}$ Division of Gastroenterology, Cheng Hsin General Hospital, Taipei, Taiwan. ${ }^{7}$ Radiation Biology Research Center, Institute for Radiological Research, Chang Gung University / Chang Gung Memorial Hospital at Linkou, Taoyuan, Taiwan.

Received: 8 April 2019 Accepted: 30 September 2019

Published online: 16 October 2019

\section{References}

1. Arteaga CL, Engelman JA. ERBB receptors: from oncogene discovery to basic science to mechanism-based cancer therapeutics. Cancer Cell. 2014; 25(3):282-303.

2. Shostak K, Chariot A. EGFR and NF-kappaB: partners in cancer. Trends Mol Med. 2015;21(6):385-93.

3. Wang J, Wang B, Chu H, Yao Y. Intrinsic resistance to EGFR tyrosine kinase inhibitors in advanced non-small-cell lung cancer with activating EGFR mutations. Onco Targets Ther. 2016:9:3711-26.

4. Lin Y, Wang $X$, Jin H. EGFR-TKI resistance in NSCLC patients: mechanisms and strategies. Am J Cancer Res. 2014;4(5):411-35.

5. Engelman JA, Zejnullahu K, Mitsudomi T, Song Y, Hyland C, Park JO, Lindeman N, Gale CM, Zhao X, Christensen J, Kosaka T, Holmes AJ, Rogers AM, Cappuzzo F, Mok T, Lee C, Johnson BE, Cantley LC, Janne PA. MET amplification leads to gefitinib resistance in lung cancer by activating ERBB3 signaling. Science. 2007;316(5827):1039-43.

6. Ninomiya K, Ohashi K, Makimoto G, Tomida S, Higo H, Kayatani H, Ninomiya T, Kubo T, Ichihara E, Hotta K, Tabata M, Maeda Y, Kiura K. MET or NRAS amplification is an acquired resistance mechanism to the third-generation EGFR inhibitor naquotinib. Sci Rep. 2018;8(1):1955.

7. Shi P, Oh YT, Zhang G, Yao W, Yue P, Li Y, Kanteti R, Riehm J, Salgia R, Owonikoko TK, Ramalingam SS, Chen M, Sun SY. Met gene amplification and protein hyperactivation is a mechanism of resistance to both first and third generation EGFR inhibitors in lung cancer treatment. Cancer Lett. 2016;380(2):494-504

8. Takezawa K, Pirazzoli V, Arcila ME, Nebhan CA, Song X, de Stanchina E, Ohashi K, Janjigian YY, Spitzler PJ, Melnick MA, Riely GJ, Kris MG, Miller VA, Ladanyi M, Politi K, Pao W. HER2 amplification: a potential mechanism of acquired resistance to EGFR inhibition in EGFR-mutant lung cancers that lack the second-site EGFRT790M mutation. Cancer Discov. 2012;2(10):922-33.

9. CsToth I, Anthoine G, Berghmans T, Mascaux C, Paesmans M, Sculier JP, Meert AP. C-erbB-3 expression in non-small cell lung cancer (NSCLC) patients treated by Erlotinib. Anticancer Res. 2011;31(1):281-5.

10. Arteaga CL. HER3 and mutant EGFR meet MET. Nat Med. 2007;13(6):675-7.

11. Yonesaka K, Hirotani K, Kawakami H, Takeda M, Kaneda H, Sakai K, Okamoto I, Nishio K, Janne PA, Nakagawa K. Anti-HER3 monoclonal antibody patritumab sensitizes refractory non-small cell lung cancer to the epidermal growth factor receptor inhibitor erlotinib. Oncogene. 2016;35(7):878-86. 
12. van Lengerich B, Agnew C, Puchner EM, Huang B, Jura N. EGF and NRG induce phosphorylation of HER3/ERBB3 by EGFR using distinct oligomeric mechanisms. Proc Natl Acad Sci U S A. 2017;114(14):E2836-E45.

13. Campbell MR, Amin D, Moasser MM. HER3 comes of age: new insights into its functions and role in signaling, tumor biology, and cancer therapy. Clin Cancer Res. 2010;16(5):1373-83.

14. Cheng CC, Chou KF, Wu CW, Su NW, Peng CL, Su YW, Chang J, Ho AS, Lin HC, Chen CG, Yang BL, Chang YC, Chiang YW, Lim KH, Chang YF. EGFR-mediated interleukin enhancer-binding factor 3 contributes to formation and survival of cancer stem-like tumorspheres as a therapeutic target against EGFR-positive non-small cell lung cancer. Lung Cancer. 2018;116:80-9.

15. Cheng CC, Liao PN, Ho AS, Lim KH, Chang J, Su YW, Chen CG, Chiang YW, Yang BL, Lin HC, Chang YC, Chang CC, Chang YF. STAT3 exacerbates survival of cancer stem-like tumorspheres in EGFR-positive colorectal cancers: RNAseq analysis and therapeutic screening. J Biomed Sci. 2018; 25(1):60.

16. Chang CC, Wu MJ, Yang JY, Camarillo IG, Chang CJ. Leptin-STAT3-G9a signaling promotes obesity-mediated breast cancer progression. Cancer Res. 2015;75(11):2375-86

17. Zulkifli AA, Tan FH, Putoczki TL, Stylli SS, Luwor RB. STAT3 signaling mediates tumour resistance to EGFR targeted therapeutics. Mol Cell Endocrinol. 2017:451:15-23.

18. Cheng CC, Chang J, Huang SC, Lin HC, Ho AS, Lim KH, Chang CC, Huang L, Chang YC, Chang YF, Wu CW. YM155 as an inhibitor of cancer stemness simultaneously inhibits autophosphorylation of epidermal growth factor receptor and G9a-mediated stemness in lung cancer cells. PLoS One. 2017; 12(8):e0182149.

19. Nakamura N, Yamauchi T, Hiramoto M, Yuri M, Naito M, Takeuchi M, Yamanaka K, Kita A, Nakahara T, Kinoyama I, Matsuhisa A, Kaneko N, Koutoku H, Sasamata M, Yokota H, Kawabata S, Furuichi K. Interleukin enhancer-binding factor 3/NF110 is a target of YM155, a suppressant of survivin. Mol Cell Proteomics. 2012;11(7):M111 013243.

20. Collins RE, Northrop JP, Horton JR, Lee DY, Zhang X, Stallcup MR, Cheng X. The ankyrin repeats of G9a and GLP histone methyltransferases are monoand dimethyllysine binding modules. Nat Struct Mol Biol. 2008;15(3):245-50.

21. Wang L, Dong $X$, Ren Y, Luo J, Liu P, Su D, Yang X. Targeting EHMT2 reverses EGFR-TKI resistance in NSCLC by epigenetically regulating the PTEN/AKT signaling pathway. Cell Death Dis. 2018;9(2):129.

22. Si W, Huang W, Zheng Y, Yang Y, Liu X, Shan L, Zhou X, Wang Y, Su D, Gao J, Yan R, Han X, Li W, He L, Shi L, Xuan C, Liang J, Sun L, Wang Y, Shang Y. Dysfunction of the reciprocal feedback loop between GATA3- and ZEB2nucleated repression programs contributes to breast cancer metastasis. Cancer Cell. 2015;27(6):822-36.

23. Tu WB, Shiah YJ, Lourenco C, Mullen PJ, Dingar D, Redel C, Tamachi A, BaAlawi W, Aman A, Al-Awar R, Cescon DW, Haibe-Kains B, Arrowsmith CH, Raught B, Boutros PC, Penn LZ. MYC interacts with the G9a histone methyltransferase to drive transcriptional repression and tumorigenesis. Cancer Cell. 2018;34(4):579-95 e8.

24. Li Y, Rogoff HA, Keates S, Gao Y, Murikipudi S, Mikule K, Leggett D, Li W, Pardee AB, Li CJ. Suppression of cancer relapse and metastasis by inhibiting cancer stemness. Proc Natl Acad Sci U S A. 2015;112(6):1839-44.

25. MacDonagh L, Gray SG, Breen E, Cuffe S, Finn SP, O'Byrne KJ, Barr MP. BBI608 inhibits cancer stemness and reverses cisplatin resistance in NSCLC. Cancer Lett. 2018;428:117-26.

26. Yang A, Qin S, Schulte BA, Ethier SP, Tew KD, Wang GY. MYC inhibition depletes cancer stem-like cells in triple-negative breast cancer. Cancer Res. 2017;77(23):6641-50

27. Poli V, Fagnocchi L, Fasciani A, Cherubini A, Mazzoleni S, Ferrillo S, Miluzio A, Gaudioso G, Vaira V, Turdo A, Gaggianesi M, Chinnici A, Lipari E, Bicciato S, Bosari S, Todaro M, Zippo A. MYC-driven epigenetic reprogramming favors the onset of tumorigenesis by inducing a stem cell-like state. Nat Commun. 2018;9(1):1024.

28. Xia J, Gill EE, Hancock RE. NetworkAnalyst for statistical, visual and networkbased meta-analysis of gene expression data. Nat Protoc. 2015;10(6):823-44.

29. Zhou L, Chen J, Li Z, Li X, Hu X, Huang Y, Zhao X, Liang C, Wang Y, Sun L, Shi M, Xu X, Shen F, Chen M, Han Z, Peng Z, Zhai Q, Chen J, Zhang Z, Yang R, Ye J, Guan Z, Yang H, Gui Y, Wang J, Cai Z, Zhang X. Integrated profiling of microRNAs and mRNAs: microRNAs located on Xq27.3 associate with clear cell renal cell carcinoma. PLoS One. 2010;5(12):e15224.
30. Agarwal V, Bell GW, Nam JW, Bartel DP. Predicting effective microRNA target sites in mammalian mRNAs. Elife. 2015;4:e05005. https://www.ncbi.nlm.nih. gov/pmc/articles/PMC4532895/.

31. Gyorffy B, Surowiak P, Budczies J, Lanczky A. Online survival analysis software to assess the prognostic value of biomarkers using transcriptomic data in non-small-cell lung cancer. PLoS One. 2013;8(12):e82241.

32. Iwasa T, Okamoto I, Suzuki M, Nakahara T, Yamanaka K, Hatashita E, Yamada Y, Fukuoka M, Ono K, Nakagawa K. Radiosensitizing effect of YM155, a novel small-molecule survivin suppressant, in non-small cell lung cancer cell lines. Clin Cancer Res. 2008;14(20):6496-504.

33. Yan X, Chen X, Liang H, Deng T, Chen W, Zhang S, Liu M, Gao X, Liu Y, Zhao C, Wang X, Wang N, Li J, Liu R, Zen K, Zhang CY, Liu B, Ba Y. miR-143 and miR-145 synergistically regulate ERBB3 to suppress cell proliferation and invasion in breast cancer. Mol Cancer. 2014;13:220.

34. Chen SF, Zhang ZY, Zhang JL. Matrine increases the inhibitory effects of afatinib on H1975 cells via the IL6/JAK1/STAT3 signaling pathway. Mol Med Rep. 2017;16(3):2733-9.

35. Ahmad R, Kumar B, Chen Z, Chen X, Muller D, Lele SM, Washington MK, Batra SK, Dhawan P, Singh AB. Loss of claudin-3 expression induces IL6/gp130/Stat3 signaling to promote colon cancer malignancy by hyperactivating Wnt/betacatenin signaling. Oncogene. 2017;36(47):6592-604.

36. Organ SL, Tsao MS. An overview of the c-MET signaling pathway. Ther Adv Med Oncol. 2011;3(1 Suppl):S7-S19.

37. Wong AL, Soo RA, Tan DS, Lee SC, Lim JS, Marban PC, Kong LR, Lee YJ, Wang LZ, Thuya WL, Soong R, Yee MQ, Chin TM, Cordero MT, Asuncion BR, Pang B, Pervaiz S, Hirpara JL, Sinha A, Xu WW, Yuasa M, Tsunoda T, Motoyama M, Yamauchi T, Goh BC. Phase I and biomarker study of OPB51602, a novel signal transducer and activator of transcription (STAT) 3 inhibitor, in patients with refractory solid malignancies. Ann Oncol. 2015; 26(5):998-1005.

38. Hong D, Kurzrock R, Kim Y, Woessner R, Younes A, Nemunaitis J, Fowler N, Zhou T, Schmidt J, Jo M, Lee SJ, Yamashita M, Hughes SG, Fayad L, PihaPaul S, Nadella MV, Mohseni M, Lawson D, Reimer C, Blakey DC, Xiao X, Hsu J, Revenko A, Monia BP, MacLeod AR. AZD9150, a next-generation antisense oligonucleotide inhibitor of STAT3 with early evidence of clinical activity in lymphoma and lung cancer. Sci Transl Med. 2015;7(314):314ra185.

39. Suresh R, Ali S, Ahmad A, Philip PA, Sarkar FH. The role of cancer stem cells in recurrent and drug-resistant lung Cancer. Adv Exp Med Biol. 2016;890:57-74.

40. Han F, He J, Li F, Yang J, Wei J, Cho WC, Liu X. Emerging roles of MicroRNAs in EGFR-targeted therapies for lung cancer. Biomed Res Int. 2015;2015: 672759.

41. Cho WC, Chow AS, Au JS. MiR-145 inhibits cell proliferation of human lung adenocarcinoma by targeting EGFR and NUDT1. RNA Biol. 2011:8(1):125-31.

42. Zhong M, Ma X, Sun C, Chen L. MicroRNAs reduce tumor growth and contribute to enhance cytotoxicity induced by gefitinib in non-small cell lung cancer. Chem Biol Interact. 2010;184(3):431-8.

43. Zhuang J, Shen L, Yang L, Huang X, Lu Q, Cui Y, Zheng X, Zhao X, Zhang D, Huang $R$, Guo H, Yan J. TGFbeta1 promotes gemcitabine resistance through regulating the LnCRNA-LET/NF90/miR-145 signaling axis in bladder cancer. Theranostics. 2017;7(12):3053-67.

44. Liu PL, Liu WL, Chang JM, Chen YH, Liu YP, Kuo HF, Hsieh CC, Ding YS, Chen WW, Chong IW. MicroRNA-200c inhibits epithelial-mesenchymal transition, invasion, and migration of lung cancer by targeting HMGB1. PLOS One. 2017;12(7):e0180844.

\section{Publisher's Note}

Springer Nature remains neutral with regard to jurisdictional claims in published maps and institutional affiliations. 